\title{
Impacto do Câncer Parental no Desenvolvimento Psicológico dos Filhos: Uma Revisão da Literatura
}

\author{
Impact of Parental Cancer in Offspring's Psychological Development: \\ Literature Review
}

\author{
Ricardo João Teixeira* \& Maria da Graça Pereira \\ Universidade do Minho, Braga, Portugal
}

\begin{abstract}
Resumo
O presente artigo engloba uma extensa revisão da literatura sobre a temática do câncer parental e as consequências pessoais e familiares desta vivência. Nesse sentido, são abordadas as principais consequências médicas e psicossociais do diagnóstico e tratamento do câncer e, usando o modelo sistémico, é descrita a influência (negativa e positiva) deste acontecimento no doente e noutros membros da família. É dado especial enfoque ao desenvolvimento psicológico dos filhos, incluindo os resultados dos estudos referentes ao stress traumático e crescimento pós-traumático, em particular no câncer da mama. São tecidas algumas implicações para a investigação nesta área, bem como sugestões para futuras investigações.

Palavras-chave: Câncer Parental; Desenvolvimento Psicológico; Stress Traumático; Crescimento Póstraumático.
\end{abstract}

\begin{abstract}
The present paper includes an extended literature review about parental cancer and personal and family consequences of such experience. Some of the most important medical and psychosocial issues regarding the diagnosis and treatment of parental cancer are presented. Using a systemic theory as the background model it addresses the influences (negative and positive) of the event in the patient and in other family members. The present paper gives special focus on the offspring's psychological development, including the results regarding traumatic stress and posttraumatic growth, particularly on breast cancer. Some implications and suggestions for future research are addressed.

Keywords: Parental Cancer; Psychological Development; Traumatic Stress; Posttraumatic Growth.
\end{abstract}

Vários estudos mostraram que acontecimentos stressantes numa família podem causar perturbações no funcionamento dos membros mais jovens (Forehand, Biggar, \& Kotchick, 1998). Actualmente, existe um foco crescente nos filhos que têm pais com uma doença física crónica. Nos últimos anos, têm surgido publicações acerca do impacto, nos filhos, da esclerose múltipla dos pais (Judicibus \& McCabe, 2004), AVC (Visser-Meily, 2005), epilepsia (Aldenkamp, Suurmeijer, Bijvoet, \& Heisen, 1990), doença inflamatória intestinal (Mukherjee \& Sloper, 2001), e VIH (Armistead, Klein, Forehand, \& Wierson, 1997). As investigações mostram que os filhos de pais com doença crónica apresentam um maior risco de desenvolver problemas emocionais e comportamentais do que os filhos de pais saudáveis. O risco para esses problemas, contudo, depende de variáveis individuais e familiares relacionadas com a própria doença (Korneluk

* Endereço para correspondência: Universidade do Minho, Escola de Psicologia, Campus de Gualtar, Braga, Portugal, 4710-057. E-mail: ricardojft@gmail.com e gracep@psi.uminho.pt
\& Lee, 1998; Rolland, 2004). Segundo Rolland (2004), as doenças crónicas variam em termos dos efeitos que têm nos filhos, ao nível do início (agudo/gradual), curso (progressivo, constante ou episódico/recorrente), resultado (fatal/diminui esperança de vida/sem consequências a longo prazo) e restrições individuais.

Independentemente do tipo de doença, as características individuais do progenitor e do filho (idade/género) assim como as características familiares (parental/relações familiares) influenciam o funcionamento psicológico dos filhos (Korneluk \& Lee, 1998).

\section{Diagnóstico e Tratamento do Câncer}

Em 2001 foram diagnosticados em Portugal 33052 novos casos de câncer. A taxa de incidência foi de 328.3/ 100000, com cerca de 55\% dos tumores malignos a serem diagnosticados no sexo masculino e $66 \%$ dos câncer diagnosticados em idades superiores a 60 anos. Dos tumores invasores em localizações comuns aos dois sexos, o câncer mais frequente foi o colorectal, seguido dos câncers da mama e do estômago (Instituto Português de Oncologia Francisco Gentil, 2001). 
O diagnóstico precoce e as melhorias no tratamento do câncer têm aumentado a taxa de sobrevivência de muitos pacientes nos últimos anos. Em 2007, a mortalidade por câncer em Portugal desceu 5\% no espaço de cinco anos (Verdecchia et al., 2007). Actualmente em Portugal metade das pessoas com câncer sobrevive mais do que cinco anos, acompanhando assim a média europeia. Apesar de a tendência ir no sentido do aumento de casos diagnosticados, associado ao envelhecimento da população, a verdade é que a tendência é também para aumentar a taxa de sobrevivência (Verdecchia et al., 2007).

Não existem dados portugueses relativos ao número de doentes com câncer que tenham filhos a viver com eles, nem que tenham filhos em idade adulta. Portugal não tem registos actualizados e fiáveis de incidência de câncer, sobretudo devido à subnotificação (Diário de Notícias, 2005).

O tratamento do câncer pode englobar cirurgia, quimioterapia, radioterapia, terapia hormonal, imunoterapia ou uma combinação destes tratamentos (Andersen \& Simonelli, 2007). O tratamento escolhido depende do tipo de câncer, da fase da doença e da condição geral do doente. Este pode experienciar problemas físicos tais como fadiga, mal-estar, perda de apetite e queixas de dor como uma consequência da doença. Os tratamentos, e sobretudo as combinações, podem ter um grande impacto no funcionamento do doente (Stephens \& Aigner, 2009).

O primeiro ano após o diagnóstico é muitas vezes caracterizado por exames diagnósticos seguidos por uma ou mais modalidades de tratamento. Após este primeiro ano, a maioria dos efeitos colaterais graves associados ao tratamento recebido desaparecem (Pitot, 2002). No entanto, resultados de investigações em pacientes com idade inferior a 65 anos revelam que, oito anos após o diagnóstico, alguns destes pacientes ainda relatam problemas físicos e limitações na vida social (Schroevers, 2003).

\section{Funcionamento Psicossocial dos Doentes}

O câncer tem um forte impacto sobre o funcionamento psicossocial do doente. Uma parte considerável dos doentes com câncer confronta-se com sintomas de depressão, ansiedade e/ou stress (Ambigga, Sherina, \& Suthahar, 2005), e habitualmente experienciam uma forte incerteza quanto ao futuro.

Existe um vasto corpo de conhecimentos que mostram que o diagnóstico e tratamento do câncer estão associados a um conjunto de dificuldades que ameaçam o bem-estar físico, psicológico e emocional dos pacientes. Assim, a dor, problemas financeiros, ansiedade, perturbações do sono, fadiga, náuseas, alterações do apetite, perda do cabelo, depressão e medo, entre muitos outros, estão entre os factores mais referidos na literatura que resultam do impacto do câncer no paciente (Chochinov, 2001; Graci, 2005; Herrstedt, 2002; Kayl \& Meyers, 2006; Rosman, 2004; Wright, Kiely, Lynch, Cull, \& Selby, 2002).
A capacidade das pessoas em se adaptarem à doença e ao seu tratamento, sob circunstâncias semelhantes, pode variar individualmente. Factores como a personalidade, o suporte social, o coping, e também variáveis relacionadas com a doença, tais como o tempo desde o diagnóstico, o tratamento e a fase da doença (Bleiker, Pouwer, van der Ploeg, Leer, \& Ader, 2000; Nordin, Berglund, Glimelius, \& Sjoden, 2001), podem afectar a capacidade de adaptação da pessoa. Por exemplo, em alguns estudos realizados concluiu-se que doentes mais jovens com câncer mostraram ter mais problemas psicossociais do que doentes mais velhos (Schroevers, 2003), e que os pais com filhos a viverem em casa preocupam-se com as consequências do acontecimento nestes, podendo experienciar sentimentos de insatisfação quanto às suas tarefas parentais (Kelley, Sikka, \& Venkatesan, 1997).

Grande parte da investigação na área da psico-oncologia tem-se centrado nas consequências psicológicas do câncer da mama em mulheres (Simon \& Robb, 2007), para além de outros grupos homogéneos de doentes com câncer, dos quais se evidenciam os câncers da próstata e testicular (Lepore \& Roberts, 2007).

Existem indícios de que o distress psicológico depende da fase da doença e do tratamento a que os doentes foram sujeitos. Maior distress associou-se à fase aguda da doença e em pessoas que tinham recebido altas doses de quimioterapia com mais efeitos secundários (Hurny et al., 1996), para além de se ter constatado uma maior prevalência de depressão em doentes numa fase avançada do que em doentes com câncer numa fase inicial (Nordin et al., 2001).

\section{Família, Doença Oncológica e Desenvolvimento Psicológico dos Filhos}

Com quase metade dos doentes diagnosticados com câncer a sobreviverem mais do que 5 anos, e sendo uma condição crónica, o câncer requer frequentemente cuidados domiciliários prolongados afectando directamente a vida familiar (Brody, 2004). Embora os estudos acerca do processo familiar de prestação de cuidados sejam ainda muito limitados, está clarificado que o câncer pode mudar a identidade familiar, os papéis, e o funcionamento diário, e que os efeitos da doença podem ser profundos e duradouros. John Rolland (2004) desenvolveu um modelo que evidencia a variação nas exigências para os membros da família nas diversas fases da doença: crise, adaptação e fase terminal. A fase da crise, o período imediatamente após o diagnóstico e durante os tratamentos, é muitas vezes encarada como um período de stress (Sales, Schulz, \& Biegel, 1992). Esta fase é caracterizada por uma ruptura abrupta no quotidiano, e o facto de ser uma doença potencialmente fatal coloca em causa os planos para o futuro da família. Assim, deverão ser antecipadas algumas decisões importantes relacionadas com tratamentos e internamentos hospitalares, e o subsequente impacto sobre as emoções e questões práticas. O período de 
Teixeira, R. J. \& Pereira, M. G. (2011). Impacto do Câncer Parental no Desenvolvimento Psicológico dos Filhos: Uma Revisão da Literatura.

tempo e a intensidade do tratamento assim como a prevalência de complicações influenciam as restrições percepcionadas pelos doentes e as exigências sobre os membros da família (Lewis, 2004). A fase de adaptação surge após o tratamento. Por vezes a situação do doente melhora ou, por outro lado, o câncer pode levar a restrições de carácter permanente e os familiares são muitas das vezes confrontados com mudanças nos padrões de estilo de vida e nos papéis. Em caso de recidiva, a família é uma vez mais confrontada com tratamentos física e emocionalmente angustiantes. Mais uma vez o medo e a incerteza surgem acerca dos efeitos dos tratamentos, e muitas vezes as famílias têm de enfrentar a possibilidade real de um pior prognóstico (Sales et al., 1992). Na fase terminal, os membros da família devem entrar em acordo no que diz respeito aos cuidados relativos à morte do doente, podendo esta ser mais ou menos previsível. Aqui, a condição física do doente piora e, geralmente, os familiares têm de assumir cuidados suplementares (Sales et al., 1992).

Cada vez mais a família está a tornar-se o principal fornecedor de cuidados informais aos doentes com câncer (Mosher \& Danoff-Burg, 2005; Thomas \& Morris, 2002). Além disso, há um crescente reconhecimento de que o diagnóstico e o tratamento do câncer, não só causa significativo desconforto para os pacientes, mas também para as suas famílias. Alguns investigadores começaram assim a ver os membros familiares como "co-sofredores” na luta contra o câncer (Matthews, Baker, \& Spillers, 2003).

Sabe-se pouco sobre como o câncer pode afectar a família (Matthews et al., 2003; Thomas \& Morris, 2002). As investigações centram-se predominantemente no impacto do câncer dos filhos nos seus pais (Goldbeck, 2001; McGrath \& Chesler, 2004), no impacto do câncer parental em filhos crianças e adolescentes (Harris \& Zakowsky, 2003; Lewis, 2004; Osborn, 2007), e esmagadoramente em cônjuges de pacientes (Case, 2004; Chen, Chu, \& Chen, 2004; Colgrove, Kim, \& Thompson, 2007; Harden, 2005; Pitceathly \& Maguire, 2003; Wagner, Bigatti, \& Storniolo, 2006). Neste sentido, Welch, Wadsworth e Compas (1996) estudaram o impacto de um diagnóstico de câncer parental sobre as crianças e adolescentes, tendo verificado que apesar de os pais não percepcionarem qualquer distress nos filhos (6-18 anos de idade) após o diagnóstico, estes tiveram cotações altas em indicadores de ansiedade, depressão e agressividade. Os adolescentes relataram mais sintomas de ansiedade e depressão do que as crianças, e os adolescentes do sexo feminino tiveram as pontuações mais altas em termos de sofrimento psíquico. É importante notar que num estudo posterior, não foram encontradas diferenças, nos mesmos sintomas, entre filhos em situação de câncer parental e filhos com pais saudáveis (Harris \& Zakowsky, 2003). Aliás, neste estudo, os adolescentes em situação de câncer parental apresentaram menos stress traumático do que os adolescentes cujos pais eram saudáveis (Harris \& Zakowsky, 2003).

Osborn (2007), numa revisão da literatura sobre o impacto do câncer parental em crianças e adolescentes en- controu, no entanto, que as crianças (entre os 3-8 anos) e adolescentes (entre os 16-18 anos) estavam em maior risco para problemas de internalização (ou seja, depressão), com as raparigas adolescentes a apresentarem um risco ainda mais acrescido (89\% a 100\% da amostra).

Embora os estudos quantitativos contribuam para o conhecimento do impacto da doença oncológica nos filhos, são contudo tendenciosos na medida em que recorrem essencialmente a instrumentos de avaliação psicopatológica, assumindo uma perspectiva de vulnerabilidade/deficiência (Mosher \& Danoff-Burg, 2005; Power \& Dell Orto, 2004). Assim, é legítimo questionar como é possível descobrir transformação e crescimento pessoal através da experiência do câncer, se os participantes são apenas avaliados no sentido de se "encaixarem” em quadros patológicos?

Conforme referido, grande parte dos estudos tem-se debruçado no impacto do câncer nos cônjuges. Os resultados empíricos indicam que muitos cônjuges experienciam distress social (i.e. isolamento) e psicológico (i.e. ansiedade, preocupação, stress, depressão), igual (Pitceathly \& Maguire, 2003; Wagner et al., 2006) ou superior ao paciente (Harden, 2005). Para além disso, o stress emocional pode continuar muito tempo após o tratamento do câncer ter terminado (Harden 2005; Northouse, Mood, Templin, Mellon, \& George, 2000; Pitceathly \& Maguire, 2003). O sofrimento psicossocial pode ser explicado pelo facto do diagnóstico de câncer muitas vezes gerar uma crise nos cônjuges cuidadores (Harden, 2005; Peleg-Oren \& Sherer, 2001) por aumentar a necessidade de lidar com o isolamento social, as despesas e as novas responsabilidades interpostas pelo diagnóstico de câncer, e simultaneamente, prestar apoio emocional à família, sem serem capazes de exprimir as suas próprias angústias.

Apesar do volume de estudos com cônjuges, um cada vez maior número de estudos começa a incluir alguns filhos (crianças, adolescentes e adultos) como participantes. Por exemplo, Lowenstein e Gilbar (2000) analisaram a percepção dos encargos entre cuidadores de idosos com câncer. Embora a maioria dos participantes fossem cônjuges, os autores também incluíram filhos em idade adulta; os cônjuges foram definidos como os principais cuidadores, e os filhos como cuidadores secundários. Os autores encontraram que os cônjuges e filhos relataram problemas emocionais na mesma magnitude, e superiores aos do doente. É interessante notar que, nesta investigação, nenhum dos filhos adultos, com idades entre os 25 e os 56 anos, estavam a viver com os pais.

Matthews et al. (2003) investigaram o impacto do câncer entre os cônjuges, filhos adultos de meia-idade, e outros membros da família (i.e. mães, pais, irmãos, etc.) A amostra incluiu também amigos dos pacientes. Apesar de limitado, uma importante contribuição deste estudo quantitativo refere-se ao facto dos cuidadores apresentarem níveis mais altos em todas as medidas de distress físico e emocional (i.e. incerteza sobre o futuro, medo de 
recorrência) do que os próprios pacientes. Além disso, o score médio dos cuidadores em termos de expectativas pessimistas, também foi significativamente superior ao dos pacientes.

Os resultados de Matthews et al. (2003) são semelhantes aos demonstrados mais recentemente por Bowman, Rose e Deimling (2006), tendo estes autores encontrado que os familiares de doentes com câncer, quer os cônjuges quer os filhos adultos, avaliaram a experiência com o câncer como mais stressante do que os pacientes, durante o diagnóstico, tratamento e remissão, tendo esta apreciação permanecido anos após a conclusão do tratamento. Neste estudo, os membros da família foram descritos como um único grupo, com idades compreendidas entre os 29 e os 86 anos, apesar de se saber que os filhos adultos e os cônjuges têm responsabilidades, obrigações e necessidades diferentes na prestação de cuidados (Bakas, Lewis, \& Parsons, 2001).

Segundo a meta-análise de Hodges, Humphris e Macfarlane (2005), sobre o distress psicológico nos familiares de pacientes com câncer, a maioria dos estudos não contempla os filhos. Dos 21 estudos analisados, em 17 os participantes foram exclusivamente cônjuges, e nos restantes 4 estudos, a esmagadora maioria dos participantes eram cônjuges, com filhos adultos (e outros cuidadores familiares, como mães, irmãs, irmãos, sobrinhos, etc.), representando menos de 5\% da amostra. Na mesma linha de investigação, Boyer et al. (2002) exploraram o distress e sintomas de stress traumático entre filhas (idades 15-71 anos) de pacientes com câncer de mama (idades 40-95 anos). Neste estudo, as filhas, que eram principalmente de meia-idade e casadas, relataram visitar a mãe uma vez por semana, e perto de $40 \%$ relataram pouco ou nenhum envolvimento nos cuidados. Ainda assim, a grande maioria das filhas (92\%) teve pensamentos sobre o diagnóstico como sendo um "trauma muito stressante", e $13 \%$ das filhas tinham sintomas consistentes com stress traumático. Cohen e Pollack (2005) expandiram o estudo da equipa de Boyer (2002), acrescentando medidas do sistema imunológico. As filhas (com idades entre 24 e os 40 anos) foram avaliadas em termos sanguíneos (ou seja, o cortisol plasmático), urinários e exames hormonais. Os resultados deste estudo revelaram que as filhas cujas mães tinham altos níveis de distress, relataram maior comprometimento do sistema imunitário. O distress das filhas foi também associado à sua percepção de sobrecarga na prestação de cuidados i.e. as filhas que se encontravam mais frequentemente com as mães (uma vez em 15 dias ou uma vez por semana) relataram maior distress psicológica e exaustão do que as filhas que se encontravam com as mães em períodos superiores a 15 dias.

\section{Câncer Parental e Stress Traumático}

O câncer é actualmente conceptualizado como um possível precipitante de sintomas da perturbação de stress pós-traumático ([PTSD], American Psychiatric Asso- ciation, 2000), quer nos doentes como nos familiares em primeiro grau. Por exemplo, Boyer et al. (2002) encontraram que $21 \%$ dos doentes com câncer da mama e $13 \%$ das respectivas filhas relataram sintomas consistentes com PTSD. A prevalência de sintomas de PTSD nas filhas foi comparável aos resultados encontrados em estudos precedentes em mulheres com câncer da mama, tendo sido descoberto que as mães com sintomas de PTSD aumentam a probabilidade de as filhas virem a manifestar estes sintomas também. Segundo a revisão efectuada por Mosher e Danoff-Burg (2005), as investigações sugerem que as familiares em primeiro grau de doentes com câncer da mama têm níveis significativamente mais elevados de pensamentos intrusivos relacionados com o câncer, e de evitamento, quando comparadas com mulheres sem história familiar de doença oncológica (Zakowski et al., 1997). Por sua vez, o estudo desenvolvido por Lindberg e Wellisch (2004) encontrou que $4 \%$ dos familiares em primeiro grau de doentes com câncer da mama mostraram níveis de sintomas consistentes com um diagnóstico de PTSD, provavelmente relacionados com o câncer e, para além disso, $7 \%$ dos familiares em primeiro grau registou sintomatologia consistente com os níveis potencialmente subclínicos de PTSD relacionado com câncer. Lerman et al. (1993), encontraram que 53\% das familiares em primeiro grau de doentes com câncer da mama teve pensamentos intrusivos, e que os níveis nestas mulheres eram comparáveis àqueles encontrados em estudos com indivíduos expostos a outros tipos de trauma.

Dada a enorme variabilidade dos níveis de distress entre familiares em primeiro grau de doentes com câncer (Erblich, Bovbjerg, \& Valdimarsdottir, 2000; Lerman et al., 1993), torna-se importante avaliar os principais preditores das suas respostas ao stress. Por exemplo, um estudo demonstrou que o evitamento constituiu um mediador parcial na relação entre constrangimentos sociais e dois tipos de distress (pensamentos intrusivos relacionados com o câncer e distress geral) entre familiares em primeiro grau de doentes com câncer da mama (Schnur, Valdimarsdottir, Montgomery, Nevid, \& Bovbjerg, 2004). Numa perspectiva sócio-cognitiva de processamento, os estudos sugerem que as reacções negativas de incompreensão ou de falta de apoio por parte de outras pessoas durante a expressão de pensamentos e de sentimentos relacionados com stress podem resultar numa tentativa de evitamento de tais pensamentos e sentimentos (Mosher \& Danoff-Burg, 2005). Assim, esta exposição inadequada ao stressor pode impedir o seu processamento em termos cognitivos, prolongando assim o distress.

A idade actual de uma mulher também pode ser associada à severidade do seu distress, conforme apontam Lerman, Kash e Stefanek (1994) no estudo com familiares em primeiro grau de doentes com câncer da mama. Os autores encontraram níveis mais elevados de pensamentos intrusivos entre as mulheres com 50 ou mais anos de idade, quando comparadas com mulheres mais novas. 
Teixeira, R. J. \& Pereira, M. G. (2011). Impacto do Câncer Parental no Desenvolvimento Psicológico dos Filhos: Uma Revisão da Literatura.

Este dado é especialmente notável à luz da relativa evidência acerca de uma associação inversa entre o distress psicológico e a adesão à mamografia entre familiares em primeiro grau de doentes com câncer (George, 2000). Segundo Baider, Ever-Hadani e De-Nour (1999), quando comparadas com mulheres que tiveram uma mãe ou uma irmã com câncer da mama, mulheres que tiveram uma história de câncer da mama em ambas as familiares revelaram ter mais pensamentos intrusivos.

A morte de um progenitor com câncer pode ser outro preditor potencial de PTSD, principalmente quando foi precedida de um longo e doloroso declínio na saúde do progenitor, assim como de prestação de cuidados a longo prazo. O estudo de Zakowski et al. (1997) revela que as filhas de doentes falecidas com câncer da mama, demonstraram ter mais pensamentos intrusivos, de evitamento, e de risco percebido. Os resultados sugerem que o risco pessoal percebido para o câncer da mama mediou o efeito da morte da progenitora nos pensamentos intrusivos e no evitamento. No estudo transversal de Erblich et al. (2000) as filhas saudáveis e cuidadoras, com história materna de câncer da mama, obtiveram valores mais elevados nas preocupações directamente relacionadas com o câncer da mama quando comparadas com filhas nãocuidadoras.

Os aspectos únicos da experiência de câncer relacionados com outros tipos de trauma, e a sobreposição entre reacções normativas ao câncer e as respostas de PTSD, complicam a interpretação das descobertas destes estudos (Kangas, Henry, \& Bryant, 2002). Por exemplo, as reacções normais de dor e sofrimento perante um diagnóstico de câncer podem ser confundidas com sintomas de PTSD porque ambos habitualmente envolvem activação, evitamento e pensamentos intrusivos (Kangas et al., 2002; Stroebe et al., 2000). Para além disso, e segundo a American Psychiatric Association (2000), a "incapacidade para lembrar aspectos importantes do trauma" (p. 468) pode ser mais apropriada para descrever o trauma discreto, do que experiências prolongadas com câncer parental. Certamente que evitar "lembretes" do trauma pode ser impossível para membros da família do doente de câncer devido, sobretudo, às sugestões externas como as consultas médicas, as prescrições medicamentosas, e os efeitos secundários dos tratamentos (Kangas et al., 2002). Ainda assim, é importante salientar que no contexto da doença oncológica a habitual definição dos sintomas re-experienciados poderá não ser a mesma que noutros contextos porque os pensamentos intrusivos podem ser preocupações futuras relacionadas com questões de saúde em vez de ruminações relativas a experiências passadas, ou seja, embora existam registos de pensamentos intrusivos em filhos de doentes oncológicos, ainda não é conhecida a sua natureza exacta (Kangas et al., 2002).

Uma explicação para as aparentes inconsistências nos estudos pode dever-se a variações entre os grupos in- cluídos, na forma como os problemas dos filhos são avaliados, juntamente com dificuldades na obtenção de amostras adequadas em termos de homogeneidade e tamanho (Watson et al., 2006). Provavelmente, alguns pais menos incapacitados pela doença tentarão evitar qualquer impacto sobre os seus filhos, enquanto outros poderão lidar pior com esta situação. Assim, pais com câncer podem não constituir um grupo homogéneo, obrigando à necessidade de avaliar a variabilidade entre sub-grupos familiares, de modo a que sejam esclarecidos os factores preditores do sofrimento dos filhos. Uma explicação potencial da variabilidade entre os casos está na natureza e gravidade da doença e do tratamento associado. Por exemplo, o recurso à quimioterapia no tratamento do câncer da mama tem-se expandido na última década. Embora este facto tenha causado um impacto positivo na sobrevida dos pacientes, pode criar alterações familiares profundas (Watson et al., 2006). O período em que as mulheres recebem quimioterapia pode ser um momento de grande tensão. As frequentes visitas ao hospital, os efeitos secundários físicos do tratamento, o aumento dos encargos financeiros através da ausência da mãe no trabalho, e o rompimento de rotinas familiares, são susceptíveis de aumentar o stress familiar. Por sua vez, as variações na doença e no tratamento, e a variabilidade dos recursos sociais, psicológicos, e de suporte da família também contribuem para que o impacto da doença nos filhos seja diferente consoante o momento da avaliação (Watson et al., 2006).

Segundo a revisão conduzida por Lewis (2004), as famílias normalmente não conhecem, compreendem, interagem, ou apoiam os filhos a lidar com o câncer da mama das mães. Há evidências de que os pais doentes podem subestimar, ou não estar cientes, de que os seus filhos têm dificuldades em lidar com o câncer parental (Nelson \& While, 2002; Welch et al., 1996). Nelson e While (2002) sublinham o impacto da inadequação parental em termos de coping como um factor de risco para as crianças. Tanto a baixa auto-estima como a má adaptação da mãe com câncer têm um impacto negativo na adaptação dos filhos, numa ampla faixa etária (8-16 anos). Lewis (2004) tem enfatizado o impacto negativo da depressão materna e da qualidade da parentalidade no funcionamento familiar e nos filhos de pessoas com câncer. Daqui resulta que os estudos sobre as perturbações dos filhos, nomeadamente sobre a PTSD, deverão avaliar as diferenças familiares em termos de comunicação familiar e de suporte parental.

Uma terceira fonte de variabilidade entre os estudos, com famílias, está nas características dos filhos, incluindo idade e sexo. Estudos anteriores sugerem que os filhos adolescentes experienciam mais problemas (Welch et al., 1996), enquanto as filhas adolescentes experenciam um maior risco (Wellisch, Gritz, Schain, Wang, \& Siau, 1992). Visser et al. (2005) relataram que as filhas adolescentes foram mais afectadas pela intensidade do 
tratamento do câncer das mães, enquanto nos filhos foi a recidiva. No entanto, a evidência sobre as diferenças de género permanece insubstancial, dado o baixo número de estudos incluindo rapazes.

\section{Câncer Parental e Crescimento Pós-Traumático}

Segundo Joseph e Linley (2008), as teorias acerca da PTSD não consideram as consequências positivas do trauma. Para os autores, é fundamental que qualquer teoria acerca de mudanças positivas não contradiga o que se sabe acerca do desenvolvimento de PTSD, mas que acomode os conhecimentos acerca desta perturbação e o crescimento, ou seja, para que se possa desenvolver a compreensão do crescimento através da adversidade, é necessário integrar em qualquer teoria do crescimento a sintomatologia de PTSD, e a forma como esses sintomas se relacionam com as mudanças psicológicas positivas.

Estudos focados no conceito de distress mostram que, efectivamente, o câncer pode ser encarado como uma transição psicossocial (Cordova, 2008), que potencialmente pode resultar em dificuldades de ajustamento, mas também num fortalecimento e crescimento pessoal. Segundo Parkes (1971, citado por Cordova, 2008), a expressão "transição psicossocial” refere-se a experiências basilares de vida que requerem que os indivíduos reestruturem as suas maneiras de olhar o mundo e os seus planos para viverem nele. De facto, esta noção está patente em vários registos sobre sobreviventes de câncer, na frequência com que elaboram atribuições positivas acerca dos seus esforços de combate à doença (Cordova, 2008; Sears, Stanton, \& Danoff-Burg, 2003). Por exemplo, um estudo qualitativo indica que algumas sobreviventes de câncer da mama experienciam um crescimento espiritual à medida que constroem um sentido de ligação com os outros ou com um poder mais elevado, encontrando assim um propósito renovado para as suas vidas (Gall \& Cornblat, 2002).

O termo "crescimento pós-traumático" (CPT) foi introduzido por Calhoun e Tedeschi (2008) para descrever mudanças positivas depois de uma doença ou outras experiências potenciadoras de stress. A experiência de doença oncológica tem tido grande enfoque no estudo dos potenciais catalisadores para uma transformação pessoal positiva, sobretudo nos doentes (Cordova, 2008; Sears et al., 2003), sabendo-se pouco acerca dos potenciais ganhos psicossociais entre outros familiares. Contudo, para Mosher, Danoff-Burg e Brunker (2006), as evidências de base sugerem que as experiências relacionadas com o câncer podem precipitar o crescimento pessoal, não só nos doentes, mas também nos seus cônjuges e filhos. Por exemplo, entre mulheres com câncer da mama, um maior CPT foi associado às idades mais novas, rendimentos mais elevados, contacto com uma pessoa de forma a expressar os benefícios da experiência de câncer, assim como uma melhor expressão emocional e stress percebido relativo ao câncer (Manne et al., 2004).
Entre os cônjuges, um CPT foi associado ao facto de se ter uma idade mais jovem, mais pensamentos intrusivos e avaliações da doença como um acontecimento traumático, assim como a um maior uso de estratégias de coping tendo em vista reapreciações positivas e o processamento emocional (Manne et al., 2004). Nesta linha de registo, Weiss (2002) verificou que grande parte dos maridos de doentes com câncer da mama refere mudanças positivas com uma intensidade pequena a média, após o diagnóstico. No âmbito da relação pais-filhos em situação de câncer parental, os estudos de Leedham e Meyerowitz (1999) assumem um carácter especialmente relevante na última década. Os autores avaliaram os benefícios derivados da experiência com câncer, tendo 93\% das filhas adultas da amostra indicado que o câncer parental tinha provocado pelo menos uma mudança positiva nas suas vidas. A tendência quase total de derivar benefícios da experiência de câncer parental na amostra deste estudo sugere que as questões de investigação podem incluir uma análise de ambas as sequelas psicossociais (positivas e negativas), e respectivos preditores, nesta população.

Um dos estudos mais relevantes neste âmbito foi conduzido por Mosher et al. (2006), tendo avaliado os preditores de CPT em filhas adultas de doentes com câncer da mama, tais como as estratégias de coping e a prestação de cuidados. Note-se que segundo o modelo teórico de Calhoun e Tedeschi (2008), os optimistas, ou pessoas que têm expectativas mais positivas relativamente aos resultados, usam estratégias de coping que promovem o CPT. Para além disso, ter tempo suficiente para processar as implicações de um diagnóstico de câncer num contexto social de bom suporte emocional é tido como principal hipótese explicativa desse crescimento. Contudo, é de salientar que nem o optimismo nem o suporte social estiverem associados ao CPT nos estudos com doentes oncológicos (Cordova, 2008; Sears et al., 2003).

Em termos comparativos, o grau de CPT numa filha cuja mãe foi diagnosticada com câncer da mama é bastante similar ao registo do mesmo CPT na própria doente (Cordova, 2008; Sears et al., 2003). No estudo de Mosher et al. (2006), as características pessoais (idade, rendimentos, instrução, estado civil, optimismo) não se correlacionaram com o CPT, embora uma melhor percepção acerca do stress relacionado com o câncer e um maior suporte social se tenham revelado fomentadores deste tipo de crescimento. Estes resultados refinam o modelo de Calhoun e Tedeschi (2008), de que o forte compromisso com um stressor, num contexto de bom suporte social, facilita o CPT.

Também consistente com o modelo de Calhoun e Tedeschi (2008) está a descoberta de que a gestão activa da emocionalidade e dos stressores associados ao câncer parental, impulsiona um maior CPT. Mais especificamente, um maior CPT gera maiores probabilidades de desenvolvimento de estratégias de planeamento de coping orientadas para a aproximação, o coping activo, a pro- 
Teixeira, R. J. \& Pereira, M. G. (2011). Impacto do Câncer Parental no Desenvolvimento Psicológico dos Filhos: Uma Revisão da Literatura.

cura de suporte social, e o processamento emocional (Mosher et al., 2006). Segundo Calhoun e Tedeschi (2008), os prestadores de cuidados recorrem a diversos recursos quando confrontados com stressores associados à doença, podendo uma tendência para a acção contribuir para o CPT.

Em Portugal, os dados preliminares da investigação de Teixeira e Pereira (2009) com filhos adultos de doentes oncológicos em tratamento quimioterapêutico, sugerem uma correlação positiva entre morbilidade psicológica (ansiedade, depressão, stress e PTSD) e as dimensões do CPT. No mesmo estudo verificou-se igualmente que o grau de dependência dos pais é uma variável importante no nível de CPT dos filhos, já que é no grupo de progenitores mais dependentes que se verificam resultados mais elevados de CPT. Estes resultados estão em concordância com o modelo de CPT proposto por Calhoun e Tedeschi (2008), em que o comprometimento com um stressor, aqui constituído pela doença e grau de dependência do progenitor, potencia uma maior percepção de CPT.

\section{Conclusão}

A revisão da investigação efectuada destaca que o diagnóstico e tratamento do câncer comportam um distress considerável aos familiares dos doentes, podendo comprometer as suas estratégias de coping. Entre os filhos adultos de meia-idade, a literatura mostra que estão em maior risco de sofrer problemas emocionais, bem como sintomas depressivos, ansiedade e PTSD. Estes filhos, que estão envolvidos nos cuidados aos seus pais, são mais propensos a apresentar uma diminuição da qualidade de vida, medo, incerteza e maior exaustão nas tarefas de cuidar.

Os estudos revistos fornecem, no entanto, uma compreensão ainda limitada da experiência de câncer parental, sobretudo do ponto de vista dos filhos em idades mais adultas e que vivem com os pais. Algumas limitações foram também identificadas no restante corpo de investigação. A maioria dos estudos sobre o impacto do câncer em membros da família tem-se centrado esmagadoramente nos cônjuges dos doentes. Dos poucos estudos que incorporaram os filhos, os resultados são agrupados sob o termo genérico de “cuidadores", impossibilitando muitas vezes a distinção da idade dos filhos uma vez que os resultados foram tratados como um único grupo. A experiência de câncer parental em filhos entre os 21 e os 35 anos de idade que vivem em casa dos progenitores (conhecida como a “geração sanduíche”) (cf. Brody, 2004), pode apresentar desafios únicos quando comparada com a experiência dos filhos que vivem na sua própria casa; filhos com uma família já constituída versus solteiros ou ainda filhos de pais idosos versus filhos de pais jovens.

A grande maioria dos estudos sobre o impacto do câncer na família tem utilizado metodologias quantitativas. Embora esta metodologia contribua para o corpo de co- nhecimentos em aspectos importantes, é inegável que a sua contribuição é limitada, pois permite apenas estudar diferenças entre grupos ou indivíduos, não permitindo uma compreensão de como a doença é vivida por cada indivíduo. Polkinghorne (2005) indica que a pesquisa quantitativa não pode ser adequada para a exploração em profundidade da plenitude da experiência de vida do participante único.

É importante também concluir que grande parte da literatura disponível é baseada numa conceptualização do câncer parental como uma experiência de sofrimento, perda e exaustão, perpetuando a ideia de que os indivíduos, face à adversidade, não são susceptíveis de atribuir um significado positivo à sua experiência, reduzindo, assim, a experiência humana à área da psicopatologia. Investigações actuais com medidas quantitativas de psicopatologia (i.e. depressão e ansiedade) reforçam a noção de que os indivíduos são incapazes de experimentar um crescimento face a uma doença fatal e, desta forma, continuam a reforçar uma perspectiva de "stress-vulnerabilidade” sobre a doença (Power \& Dell Orto, 2004). Contudo, a psicologia positiva tem defendido que as pessoas são únicas, podendo construir significados originais das suas experiências. No âmbito deste trabalho, embora o diagnóstico de uma doença fatal como o câncer possa trazer perdas, é também verdade que este diagnóstico pode ter um potencial de transformação. Como fazem notar Power e Dell Orto (2004), a doença pode ser redefinida não só como uma experiência de perda e sofrimento, mas também como uma oportunidade para superar os enormes desafios que a doença acarreta. Estes resultados positivos face à adversidade estão cada vez mais reconhecidos na literatura empírica, dada a atenção que o CPT tem merecido na investigação com implicações ao nível da intervenção clínica (Calhoun \& Tedeschi, 2007).

\section{Referências}

Aldenkamp, A. P., Suurmeijer, T. P., Bijvoet, M. E., \& Heisen, T. W. (1990). Emotional and social reactions of children to epilepsy in a parent. Family Practice, 7, 110-115.

Ambigga, D. K., Sherina, M. S., \& Suthahar, A. (2005). Depression and anxiety among family caregivers of cancer patients in an oncology clinic. Malaysian Journal of Psychiatry, 13, 35-42.

American Psychiatric Association. (2000). Diagnostic and statistical manual of mental disorders ( $4^{\text {th }} \mathrm{Rev}$. Ed.). Washington, DC: Author.

Andersen, B. L., \& Simonelli, L. E. (2007). Cancer: General. In S. Ayers, A. Baum, C. McManus, S. Newman, K. Wallston, J. Weinman, et al. (Eds.), Cambridge Handbook of Psychology, health and Medicine ( $2^{\text {nd }}$ ed., pp. 584-591). New York: Cambridge University Press.

Armistead, L., Klein, K., Forehand, R., \& Wierson, M. (1997). Disclosure of parental HIV infection to children in the families of men with hemophilia: Description, outcomes, and the role of family processes. Journal of Family Psychology, 11, 49-61. 
Baider, L., Ever-Hadani, P., \& De-Nour, A. K. (1999). Psychological distress in healthy women with familial breast cancer: Like mother, like daughter? International Journal of Psychiatry in Medicine, 29, 411-420.

Bakas, T., Lewis, R. R., \& Parsons, E. (2001). Caregiving tasks among family caregivers of patients with lung cancer. Oncology Nursing Forum, 28, 847-854.

Bleiker, E. M. A., Pouwer, F., van der Ploeg, H. M., Leer, J. W. H., \& Ader, H. J. (2000). Psychological distress two years after diagnosis of breast cancer: Frequency and prediction. Patient Education and Counseling, 40, 209-217.

Bowman, K. F., Rose, J. H., \& Deimling, G. T. (2006). Appraisal of the cancer experience by family members and survivors in long-term survivorship. Psycho-Oncology, 15, 834-845.

Boyer, B. A., Bubel, D., Jacobs, S. R., Knolls, M. L., Harwell, V. D., Goscicka, M., et al. (2002). Posttraumatic stress in women with breast cancer and their daughters. American Journal of Family Therapy, 30, 323-338.

Brody, E. M. (2004). Women in the middle: Their parent-care years. New York: Springer.

Calhoun, L., \& Tedeschi, R. (2007). Crescimento pós-traumático em intervenções clínicas cognitivo-comportamentais. In V. E. Caballo (Ed.), Manual para o tratamento cognitivo comportamental dos transtornos psicológicos da actualidade (pp. 29-47). São Paulo, SP: Santos.

Calhoun, L., \& Tedeschi, R. (2008). The paradox of struggling with trauma: Guidelines for practice and directions for research. In S. Joseph \& P. A. Linley (Eds.), Trauma, recovery, and growth: Positive Psychological perspectives on posttraumatic stress (pp. 325-337). Hoboken, NJ: John Wiley \& Sons.

Case, P. (2004). Surviving cancer and family costs: Examining the needs of spouses. Illness Crisis \& Loss, 12, 126-138.

Chen, M., Chu, L., \& Chen, H. (2004). Impact of cancer patients' quality of life on that of spouse caregivers. Supportive Care in Cancer, 12, 469-475.

Chochinov, H. M. (2001). Depression in cancer patients. Lancet Oncology, 2, 499-505.

Cohen, M., \& Pollack, S. (2005). Mothers with breast cancer and their adult daughters: The relationship between mothers' reaction to breast cancer and their daughters' emotional and neuroimmune status. Psychosomatic Medicine, 67, 64-71.

Colgrove, L. A., Kim, Y., \& Thompson, N. (2007). The effect of spirituality and gender on the quality of life of spousal caregivers of cancer survivors. Annals Behavioral Medicine, 33, 90-98.

Cordova, M. J. (2008). Facilitating posttraumatic growth following cancer. In S. Joseph \& P. A. Linley (Eds.), Trauma, recovery, and growth: Positive Psychological perspectives on posttraumatic stress (pp. 185-205). Hoboken, NJ: John Wiley \& Sons.

Diário de Notícias (2005). Registos oncológicos nacionais atrasados. Retrieved June 1, 2009, from http://dn.sapo.pt/inicio/ interior.aspx?content_id=607836

Erblich, J., Bovbjerg, D. H., \& Valdimarsdottir, H. B. (2000). Looking forward and back: Distress among women at familial risk for breast cancer. Annals of Behavioral Medicine, 22, 53-59.

Forehand, R., Biggar, H., \& Kotchick, B. A. (1998). Cumulative risk across family stressors: Short- and longterm effects for adolescents. Journal of Abnormal Child Psychology, 26, 119-128.
Gall, T. L., \& Cornblat, M. W. (2002). Breast cancer survivors give voice: A qualitative analysis of spiritual factors in longterm adjustment. Psycho-Oncology, 11, 524-535.

George, S. A. (2000). Barriers to breast cancer screening: An integrative review. Health Care for Women International, 21, 53-65.

Goldbeck, L. (2001). Parental coping with the diagnosis of childhood cancer: Gender effects, dissimilarity within couples, and quality of life. Psycho-Oncology, 10, 325-335.

Graci, G. (2005). Pathogenesis and management of cancer-related insomnia. Journal of Supportive Oncology, 3, 349-359.

Harden, J. (2005). Developmental life stage and couples' experiences with prostate cancer: A review of the literature. Cancer Nursing, 28, 85-98.

Harris, C. A., \& Zakowsky, S. (2003). Comparisons of distress in adolescents of cancer patients and controls. PsychoOncology, 12,173-182.

Herrstedt, J. (2002). Nausea and emesis: Still an unresolved problem in cancer patients? Supportive Care in Cancer, 10, 85-87.

Hodges, L. J., Humphris, G. M., \& Macfarlane, G. (2005). A meta-analytical investigation of the relationship between the psychological distress of cancer patients and their carers. Social Science \& Medicine, 60, 1-12.

Hurny, C., Bernard, J., Coates, A. S., Castiglione-Gertsch, N., Peterson, H. F., Gelber, R. D., et al. (1996). Impact of adjuvant therapy on quality of life in women with node-positive operable breast cancer. International Breast Cancer Study Group. Lancet, 347, 1279-1284.

Instituto Português de Oncologia Francisco Gentil. (2001). Registo Oncológico Nacional 2001. Porto, Portugal: Autor.

Joseph, S., \& Linley, P. A. (2008). Reflections on theory and practice in trauma, recovery, and growth: A paradigm shift for the field of traumatic stress. In S. Joseph \& P. A. Linley (Eds.), Trauma, recovery, and growth: Positive Psychological perspectives on posttraumatic stress (pp. 339-356). Hoboken, NJ: John Wiley \& Sons.

Judicibus, M. A., \& McCabe, M. P. (2004). The impact of parental multiple sclerosis on the adjustment of children and adolescents. Adolescence, 39, 551-569.

Kangas, M., Henry, J. L., \& Bryant, R. A. (2002). Posttraumatic stress disorder following cancer: A conceptual and empirical review. Clinical Psychology Review, 22, 499-524.

Kayl, A. E., \& Meyers, C. A. (2006). Side-effects of chemotherapy and quality of life in ovarian and breast cancer patients. Current Opinion in Obstetrics \& Gynecology, 18, 24-28.

Kelley, S. D. M., Sikka, A., \& Venkatesan, S. (1997). A review of research on parental disability: Implications for research and counseling practice. Rehabilitation Counseling Bulletin, 41, 105-121.

Korneluk, Y. G., \& Lee, C. M. (1998). Children's adjustment to parental physical illness. Clinical Child and Family Psychology Review, 1, 179-193.

Leedham, B., \& Meyerowitz, B. E. (1999). Responses to parental cancer: A clinical perspective. Journal of Clinical Psychology in Medical Settings, 6, 441-461.

Lepore, S. J., \& Roberts, K. J. (2007). Cancer: Prostate. In S. Ayers, A. Baum, C. McManus, S. Newman, K. Wallston, J. Weinman, et al. (Eds.), Cambridge Handbook of Psychology, health and Medicine ( $2^{\text {nd }}$ ed., pp. 607-609). New York: Cambridge University Press. 
Teixeira, R. J. \& Pereira, M. G. (2011). Impacto do Câncer Parental no Desenvolvimento Psicológico dos Filhos: Uma Revisão da Literatura.

Lerman, C., Daly, M., Sands, C., Balshem, A., Lustbader, E., Heggan, T., et al. (1993). Mammography adherence and psychological distress among women at risk for breast cancer. Journal of the National Cancer Institute, 85, 1074-1080.

Lerman, C., Kash, K., \& Stefanek, M. (1994). Younger women at increased risk for breast cancer: Perceived risk, psychological well-being, and surveillance behavior. Journal of the National Cancer Institute. Monographs, 16, 171-176.

Lewis, F. M. (2004). Family-focused oncology nursing research. Oncology Nursing Forum, 31, 288-292.

Lindberg, N. M., \& Wellisch, D. K. (2004). Identification of traumatic stress reactions in women at increased risk for breast cancer. Psychosomatics, 45, 7-16.

Lowenstein, A., \& Gilbar, O. (2000). The perception of caregiving burden on the part of elderly cancer patients, spouses, and adult children. Families, System, and Health, 18, 337-346.

Manne, S., Ostroff, J., Winkel, G., Goldstein, L., Fox, K., \& Grana, G. (2004). Posttraumatic growth after breast cancer: Patient, partner, and couple perspectives. Psychosomatic Medicine, 66, 442-454.

Matthews, B. A., Baker, F., \& Spillers, R. L. (2003). Family caregivers and indicators of cancer-related distress. Psychology, Health, \& Medicine, 8, 45-56.

McGrath, P., \& Chesler, M. (2004). Father's perspective on the treatment for pediatric hematology: Extending the findings. Issues in Comprehensive Pediatric Nursing, 2, 39-61.

Mosher, C. E., \& Danoff-Burg, S. (2005). Psychosocial impact of parental cancer in adulthood: A conceptual and empirical review. Clinical Psychology Review, 25, 365-382.

Mosher, C. E., Danoff-Burg, S., \& Brunker, B. (2006). Posttraumatic growth and psychosocial adjustment of daughters of breast cancer survivors. Oncology Nursing Forum, 33, 543-551.

Mukherjee, S., \& Sloper, P. (2001). Understanding the impact of inflammatory bowel disease on parents and their children. New York: University of York.

Nelson, E., \& While, D. (2002). Children's adjustment during the first year of a parent's cancer diagnosis. Journal of Psychosocial Oncology, 20, 15-36.

Nordin, K., Berglund, G., Glimelius, B., \& Sjoden, P.-O. (2001). Predicting anxiety and depression among cancer patients: A clinical model. European Journal of Cancer, 37, 376-384.

Northouse, L. L., Mood, D., Templin, T., Mellon, S., \& George, T. (2000). Couples' patterns of adjustment to colon cancer. Social Science \& Medicine, 50, 271-284.

Osborn, T. (2007). The psychosocial impact of parental cancer on children and adolescents: A systematic review. PsychoOncology, 16, 101-126.

Peleg-Oren, N., \& Sherer, M. (2001). Cancer patients and their spouses: Gender and its effect on psychological and social adjustment. Journal of Health Psychology, 6, 329-338.

Pitceathly, C., \& Maguire, P. (2003). The psychological impact of cancer on patients' partners and other key relatives: A review. European Journal of Cancer, 39, 1517-1524.

Pitot, H. C. (2002). Fundamentals of Oncology. New York: Marcel Dekker.

Polkinghorne, D. E. (2005). Language and meaning: Data collection in qualitative research. Journal of Counseling Psychology, 52, 137-145.

Power, W. E., \& Dell Orto, A. E. (2004). Families living with chronic illness and disability: Interventions, challenges, and opportunities. New York: Springer.
Rolland, J. S. (2004). Families and chronic illness: An integrative model. In D. R. Catherall (Ed.), Handbook of stress, trauma, and the family (pp. 89-116). New York: Brunner-Routledge.

Rosman, S. (2004). Cancer and stigma: Experience of patients with chemotherapy- induced alopecia. Patient Education and Counseling, 52, 333-339.

Sales, E., Schulz, R., \& Biegel, D. (1992). Predictors of strain in families of cancer patients: A review of the literature. Journal of Psychosocial Oncology, 10, 1-26.

Schnur, J. B., Valdimarsdottir, H. B., Montgomery, G. H., Nevid, J. S., \& Bovbjerg, D. H. (2004). Social constraints and distress among women at familial risk for breast cancer. Annals of Behavioral Medicine, 28, 142-148.

Schroevers, M. J. (2003). Short- and long-term adaptation to cancer: A comparison of patients with the general population. Unpublished master's thesis, University of Groningen, Netherlands.

Sears, S. R., Stanton, A. L., \& Danoff-Burg, S. (2003). The yellow brick road and the emerald city: Benefit finding, positive reappraisal coping, and posttraumatic growth in women with early-stage breast cancer. Health Psychology, 22, 487-497.

Simon, A., \& Robb, K. (2007). Cancer: Breast. In S. Ayers, A. Baum, C. McManus, S. Newman, K. Wallston, J. Weinman, et al. (Eds.), Cambridge Handbook of Psychology, health and Medicine ( ${ }^{\text {nd }}$ ed., pp. 577-580). New York: Cambridge University Press.

Stephens, F. O., \& Aigner, K. R. (2009). Basics of Oncology. New York: Springer.

Stroebe, M., van Son, M., Stroebe, W., Kleber, R., Schut, H., \& van den Bout, J. (2000). On the classification and diagnosis of pathological grief. Clinical Psychology Review, 20, 57-75.

Teixeira, R. J., \& Pereira, M. G. (2009). Variáveis familiares e psicológicas em filhos adultos de doentes oncológicos. In Livro de Actas do I Congresso Luso-Brasileiro de Psicologia da Saúde [CD-ROM]. Algarve, Portugal: Universidade do Algarve.

Thomas, C., \& Morris, S. M. (2002). Informal carers in contexts. European Journal of Cancer Care, 11, 178-182.

Verdecchia, A., Franciscia, S., Brenner, H., Gatta, G., Micheli, A., Mangone, L., et al. (2007). Recent cancer survival in Europe: A 2000-02 period analysis of EUROCARE-4 data 2007. The Lancet Oncology, 8, 784-796.

Visser, A., Huizinga, G. A., Hoekstra, H. J., van der Graaf, W. T., Klip, E. C., Pras, E., et al. (2005). Emotional and behavioural functioning of children of a parent diagnosed with cancer: A cross-informant perspective. PsychoOncology, 14, 746-758.

Visser-Meily, M. (2005). Children's adjustment to a parent's stroke: Determinants of health status and psychological problems, and the role of support from the rehabilitation team. Journal of Rehabilitation Medicine, 37, 236-241.

Wagner, C. D., Bigatti, S. M., \& Storniolo, A. M. (2006). Quality of life of husbands of women with breast cancer. PsychoOncology, 15, 109-120.

Watson, M., James-Roberts, I., Ashley, S., Tilney, C., Brougham, B., Edwards, L., et al. (2006). Factors associated with emotional and behavioural problems among school age children of breast cancer patients. British Journal of Cancer, 94, 43-50.

Weiss, T. (2002). Posttraumatic growth in women with breast cancer and their husbands: An intersubjective validation study. Journal of Psychosocial Oncology, 20, 65-80. 
Welch, A. S., Wadsworth, M. E., \& Compas, B. E. (1996). Adjustment of children and parental cancer: Parents' and children's perspective. Cancer, 77, 1409-1418.

Wellisch, D. K., Gritz, E. R., Schain, W., Wang H.-J., \& Siau, J. (1992). Psychological functioning of daughters of breast cancer patients, Part II: Characterizing the distressed daughter of the breast cancer patient. Psychosomatics, 33, 171-179.

Wright, E. P., Kiely, M. A., Lynch, P., Cull, A., \& Selby, P. (2002). Social problems in Oncology. British Journal of Cancer, 87, 1099-1104.

Zakowski, S. G., Valdimarsdottir, H. B., Bovbjerg, D. H., Borgen, P., Holland, J., Kash, K., et al. (1997). Predictors of intrusive thoughts and avoidance in women with family histories of breast cancer. Annals of Behavioral Medicine, 19, 362-369. 